\title{
ASYMPTOTIC BEHAVIOUR OF THE INVERSE OF A LAPLACE TRANSFORM
}

\author{
T. E. HULL AND C. FROESE
}

1. Introduction. There are many problems, particularly in circuit theory, where the inverse of a Laplace transform is required but only for large values of the independent variable. For example (2, p. 66) the Laplace transform

$$
i(s)=\int_{0}^{\infty} e^{-s t} I(t) d t
$$

of the current function for a semi-infinite cable under certain conditions turns out to be

$$
i(s)=\frac{s^{\frac{1}{2}}}{1+s} .
$$

To find the behaviour of $I(t)$ for large $t$ we can proceed as follows. We first expand $i(s)$ in powers of $s$ to obtain

$$
i(s)=s^{\frac{1}{2}}-s^{3 / 2}+s^{5 / 2}-\ldots
$$

We then find the correct asymptotic expansion

$$
I(t) \sim-\left(\frac{2}{\pi}\right)^{\frac{1}{2}}\left[(2 t)^{-3 / 2}+1 \cdot 3(2 t)^{-5 / 2}+1 \cdot 3 \cdot 5(2 t)^{-7 / 2}+\ldots\right]
$$

by using formally, for all $\alpha$, the result

$$
\int_{0}^{\infty} e^{-s t} \frac{t^{\alpha-1}}{\Gamma(\alpha)} d t=s^{-\alpha}
$$

which actually holds only for $\Re(\alpha)>0$.

Heaviside (6) developed this procedure in a somewhat different form and used it to solve a large number of practical problems. But it was only many years later with the development of Laplace transform theory that his methods were established on a rigorous basis $(\mathbf{1} ; \mathbf{2})$.

More recently Doetsch (4) has given a number of rather loosely connected theorems which enable one to handle more complicated transforms than the one given above. He discusses in detail the cases when the transform function behaves near the origin like $s^{-\alpha} \log ^{n} s$, all $\alpha$ and $n=0,1,2, \ldots$

It is the purpose of this paper to show how his methods can be improved and generalized so as to give results when the transform function behaves near the origin like, for example, $s^{-\alpha} e^{-\beta / s}$ or $s^{-\alpha} / \log s$, all $\alpha, \beta$.

The main results are contained in two theorems-one about properties of the inversion integral and one about comparison functions. Some of the for-

Received May 31, 1954. This investigation was carried out while Miss Froese held a National Research Council Scholarship. 
mulas needed for applications are collected in a table and a few examples are treated briefly at the end.

2. Properties of the inversion integral. The Laplace transform $f(s)$ of a function $F(t)$ is defined in the right half plane $\Re(s)>s_{0}$ by

$$
f(s)=\int_{0}^{\infty} e^{-s t} F(t) d t
$$

if this integral exists for $\Re(s)>s_{0}$. Under some circumstances the inversion integral

$$
F(t)=\frac{1}{2 \pi i} \int_{a-i_{\infty}}^{a+i_{\infty}} e^{s t} f(s) d s,
$$

is valid when $a>s_{0}$. In what follows all such integrals will be considered Lebesgue integrals or principal values of Lebesgue integrals. For example, the inversion integral is to be taken as a principal value so that

$$
\int_{a-i \infty}^{a+i_{\infty}} \text { stands for } \lim _{R \rightarrow \infty} \int_{a-i R}^{a+i R} \text {. }
$$

We shall begin with a theorem about certain properties of the inversion integral. Our plan is to first impose a few mild restrictions on the transform function. To avoid unnecessary complications we restrict ourselves to considering singularities only at the origin. We then show that the path of integration can be altered in such a way that the asymptotic behaviour of the integral is determined by its contribution from a small portion of the contour around the origin. Obvious generalizations will appear when we consider examples in $\$ 4$.

THEOREM 1. If

(i) $f(s)$ is an analytic function of $s$, regular for $|\arg s| \leqslant \psi$ for some $\psi$ where $\frac{1}{2} \pi<\psi \leqslant \pi$, except perhaps for a singularity at the origin, ${ }^{1}$

(ii) $e^{k s} f(s) \rightarrow 0$ uniformly as $|s| \rightarrow \infty$ in the sectors $\frac{1}{2} \pi \leqslant|\arg s| \leqslant \psi$, for some finite $k$,

$$
F(t)=\frac{1}{2 \pi i} \int_{a-i_{\infty}}^{a+i_{\infty}} e^{s t} f(s) d s
$$

exists for $t>0$, at least as a principal value, for some finite $a>0$, and

(iv) $f(s) \rightarrow 0$ as $|s| \rightarrow \infty$ in the strip $0 \leqslant \Re(s) \leqslant a$, then

$$
F(t)=\frac{1}{2 \pi i} \int_{C} e^{s t} f(s) d s
$$

where $C$ is the heavy contour shown in Fig. 1 and where the integral is taken to be a principal value; moreover,

$$
\frac{1}{2 \pi i} \int_{C^{\prime}} e^{s t} f(s) d s=o\left(e^{-\epsilon t}\right),
$$

$$
t \rightarrow \infty
$$

for some $\epsilon>0$, where $C^{\prime}$ is the part of $C$ for which $|s| \geqslant r>0$.

In case $f(s)$ is not single-valued we shall restrict our attention to one of its branches. 
For the proof we notice first that, because of (i), the integral around the closed contour in Fig. 1 is zero. The first result then follows from (iii) as soon as we can show that the contribution when $|s|=R$ to the integral around the closed contour approaches zero as $R \rightarrow \infty$ while $t>k$.

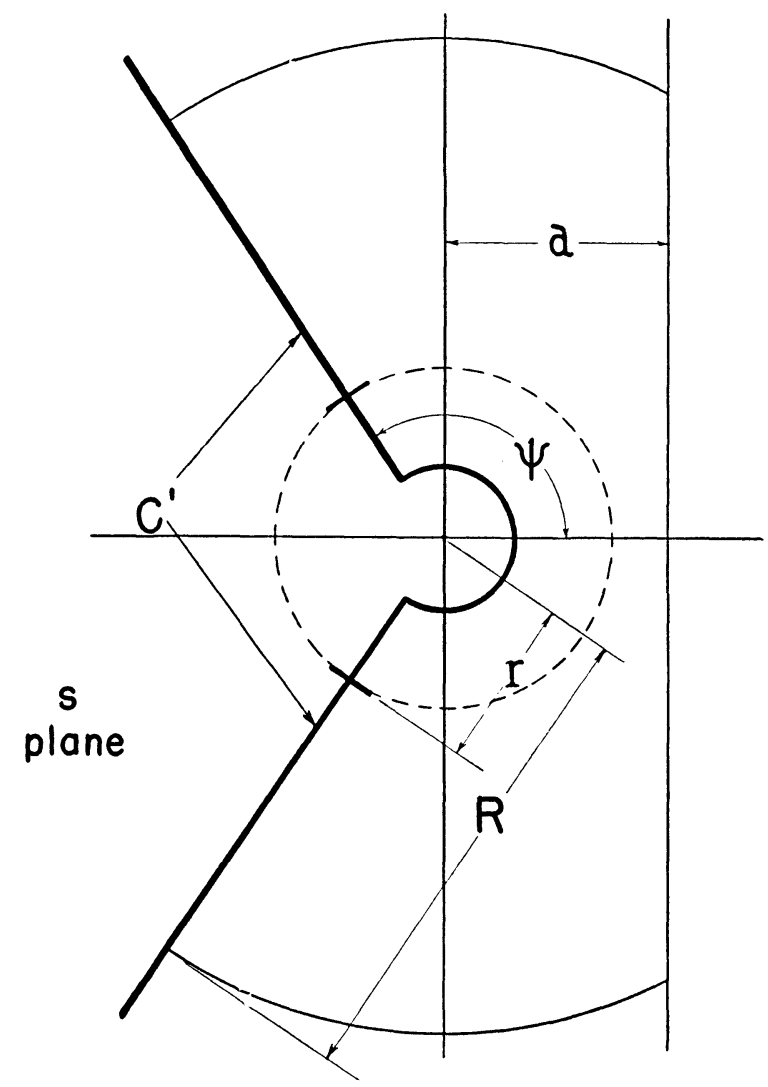

Fig. 1. $C$ is the heavy contour. $C^{\prime}$ is the portion of $C$ with $|s| \geqslant r>0$.

The parts of this contribution in the strip $0 \leqslant \Re(s) \leqslant a$ approach zero because of (iv); the remaining parts approach zero because, using (ii), the absolute value $I$ of the part in the second quadrant is

$$
I \leqslant \frac{R \delta}{2 \pi} \int_{\pi / 2}^{\psi} e^{R t \cos \theta} e^{-k R \cos \theta} d \theta
$$

where $\delta \rightarrow 0$ as $R \rightarrow \infty$. Hence

$$
\begin{aligned}
I & \leqslant \frac{\delta R}{2 \pi} \int_{\pi / 2}^{\psi} e^{R(t-k)(1-2 \theta / \pi)} d \theta \\
& =\frac{\delta}{4(t-k)}\left[1-e^{R(t-k)(1-2 \psi / \pi)}\right] \\
& \rightarrow 0
\end{aligned}
$$$$
R \rightarrow \infty \text { and } t>k,
$$ 
and similarly for the part in the third quadrant. The second result follows because

for $\epsilon=-r \cos \psi>0$.

$$
\begin{aligned}
\left|\frac{1}{2 \pi i} \int_{C^{\prime}} e^{s t} f(s) d s\right| & \leqslant \frac{\delta}{\pi}\left|\int_{r}^{\infty} e^{(t-k) u \cos \psi} d u\right| \\
& =\frac{\delta e^{-k r \cos \psi}}{-(t-k) \cos \psi}\left(e^{t r \cos \psi}\right) \\
& =o\left(e^{-t t}\right)
\end{aligned}
$$$$
\text { as } t \rightarrow \infty \text {, }
$$

3. Comparison functions. We shall now consider a theorem about certain standard transforms which will be used later for comparison with a given transform. We shall prove

THEOREM 2. If

$$
F(t)=\left\{\begin{array}{cl}
0, & 0<|t|<1 \\
\frac{1}{2} \Phi(t), & |t|=1, \\
\Phi(t), & |t|>1,
\end{array}\right.
$$

where $\Phi(t)$ is any function from the second column of the table, then the Laplace transform of $F(t)$ exists for $\Re(s)>0$ and can be represented for all $s$ by

$$
f(s)=\phi(s)+\text { an entire function, }
$$

where $\phi(s)$ is the function from the table corresponding to the $\Phi(t)$ being considered. Moreover $f(s)$ satisfies the conditions of Theorem 1 (and the integral defined in (iii) is the $F(t)$ given above).

We shall first prove the theorem for case 1 of the table and then show how this result can be generalized to give the remaining results.

Case 1. Here

so that $f(s)$ exists and

$$
\Phi(t)=\frac{t^{\alpha-1}}{\Gamma(\alpha)}
$$

$$
f(s)=\int_{1}^{\infty} e^{-s t} \frac{t^{\alpha-1}}{\Gamma(\alpha)} d t, \quad \Re(s)>0 .
$$

For $\Re(\alpha)>0$ we can write

$$
\begin{aligned}
f(s) & =\int_{0}^{\infty} e^{-s t} \frac{t^{\alpha-1}}{\Gamma(\alpha)} d t-\int_{0}^{1} e^{-s t} \frac{t^{\alpha-1}}{\Gamma(\alpha)} d t & \\
& =s^{-\alpha}+\text { an entire function, } & \Re(s)>0 .
\end{aligned}
$$

When $\Re(\alpha) \leqslant 0$ and $\alpha \neq 0,-1,-2, \ldots$, we choose an integer $n$ so that $-n<\Re(\alpha)<-n+1$, perform $n$ integrations by parts and obtain

$$
\begin{array}{rlrl}
f(s)= & e^{-s}\left(\frac{1}{\Gamma(\alpha+1)}+\frac{s}{\Gamma(\alpha+2)}+\ldots \frac{s^{n-1}}{\Gamma(\alpha+n)}\right) \\
& +\frac{s^{n}}{\Gamma(\alpha+n)} \int_{1}^{\infty} e^{-s t} t^{\alpha+n-1} d t, & \Re(s)>0 .
\end{array}
$$




\section{TABLE OF COMPARISON FUNCTIONS}

$\alpha, \beta$ are complex numbers, $n$ is a positive integer.

\begin{tabular}{l|c|c|c}
\hline & $\phi(s)$ & $\Phi(t)$ & Restrictions \\
\hline Case 1 & $\frac{1}{s^{\alpha}}$ & $\frac{t^{\alpha-1}}{\Gamma(\alpha)}$ & $\alpha \neq 0,-1,-2, \ldots$ \\
\hline Case 2a & $\frac{\log ^{n} s}{s^{\alpha}}$ & $\left(-\frac{d}{d \alpha}\right)^{n} \frac{t^{\alpha-1}}{\Gamma(\alpha)}$ & $\alpha \neq 0,-1,-2, \ldots$ \\
\hline $\begin{array}{l}\text { Case 2b } \\
(n=1)\end{array}$ & $\frac{\log s}{s^{\alpha}}$ & $-\frac{t^{\alpha-1}}{\Gamma(\alpha)}\left(\log t-\frac{\Gamma^{\prime}(\alpha)}{\Gamma(\alpha)}\right)$ & $\alpha \neq 0,-1,-2, \ldots$ \\
\hline $\begin{array}{l}\text { Case 2c } \\
(n=1) \\
(\alpha \rightarrow 0,-1, \ldots)\end{array}$ & $\frac{\log s}{s^{\alpha}}$ & $\frac{(-1)^{\alpha+1}(-\alpha) ! t^{\alpha-1}}{s^{-\beta / s}}$ & $\alpha=0,-1,-2, \ldots$ \\
\hline $\begin{array}{l}\text { Case 3 } \\
\text { Case } 4\end{array}$ & $\frac{e^{-\beta / s} \log ^{n} s}{s^{\alpha}}$ & $\frac{\left(\frac{t}{\beta}\right)^{\frac{1}{2}(\alpha-1)} J_{\alpha-1}(2 \sqrt{\beta t})}{\left(-\frac{d}{d \alpha}\right)^{n}\left(\frac{t}{\beta}\right)^{\frac{1}{2}(\alpha-1)}}$ & none \\
\hline Case 5 & $\frac{1}{s^{\alpha} \log s}-\frac{1}{s-1}$ & $\int_{\alpha-1}^{\alpha+\infty} \frac{t^{u-1}}{\Gamma(u)} d u-e^{t}$ & none \\
\hline
\end{tabular}

The first term is an entire function, and the integral in the second term can be treated as above in (4). We then obtain

$$
f(s)=s^{-\alpha}+\text { an entire function, } \quad \Re(s)>0 .
$$

We now change the path of integration in the $t$-plane from the ray $(1, \infty)$ to the contour consisting of the line segment $(1,2)$, the circular arc $\left(2,2 e^{i \theta}\right)$ and the ray $\left(2 e^{i \theta}, \infty e^{i \theta}\right)$. We obtain in this way the analytic continuation of $f(s)$ into the half-plane $\Re\left(s e^{i \theta}\right)>0$. (To obtain the continuation into the lower half-plane we let $\theta$ increase to $\frac{1}{2} \pi$ and for the upper half-plane we let $\theta$ decrease to $-\frac{1}{2} \pi$.) Equation (3) becomes

$$
\begin{aligned}
& f(s)=\int_{1}^{2} e^{-s t} \frac{t^{\alpha-1}}{\Gamma(\alpha)} d t+2^{\alpha} \int_{0}^{\theta} e^{-2 s e^{i \phi}} \frac{e^{i \alpha \phi}}{\Gamma(\alpha)} d \phi+e^{\alpha i \theta} \int_{2}^{\infty} e^{-s u e^{i \theta}} \frac{u^{\alpha-1}}{\Gamma(\alpha)} d u \\
&=s^{-\alpha}+\text { an entire function, } \\
& \Re\left(s e^{i \theta}\right)>0,
\end{aligned}
$$

by an argument similar to that used in (4) and (5). Thus $f(s)$ has the representation required by the theorem in case 1 .

That $f(s)$ satisfies condition (i) of Theorem 1 follows from the representation which has been established. 
Condition (iii) (and the fact that the integral defined in (iii) is the original $F(t)$ ) follows from the absolute convergence of (3) and the fact that $F(t)$ is of bounded variation in every finite interval and continuous except at $t=1$ (4, p. 212).

For both conditions (ii) and (iv) we have first to show that $f(s) \rightarrow 0$ as $s \rightarrow \pm i \infty$. If we put $s=-i y(y>0)$ then in (6) we can choose $\theta$, independently of $y$ and where $0<\theta<\pi$, so that the middle term is less in absolute value than any $\epsilon>0$. The third term then approaches zero as $y \rightarrow \infty$ because $\Re\left(s e^{i \theta}\right)>0$, and so does the first term because of the Riemann-Lebesgue theorem. Putting $s=i y(y>0)$ leads to a similar result.

The remainder of condition (iv) follows from the absolute convergence of (3) and the Riemann-Lebesgue theorem.

For the remainder of condition (ii) we first choose $\theta$ so that all rays in the sector $\frac{1}{2} \pi<\arg s \leqslant \psi \leqslant \pi$ enter the half-plane $\Re\left(s e^{i \theta}\right)>0$. The third term in (6) then approaches zero as $|s| \rightarrow \infty$ in this sector; the first and second terms are easily shown to be $O[\exp (-2 \Re(s))]$ as $|s| \rightarrow \infty$ in the sector. Similar results hold in the sector $-\pi \leqslant \psi \leqslant \arg s<-\frac{1}{2} \pi$ and (ii) follows for any $k>2$.

Thus Theorem 2 has been proven for case 1 of the table. From our point of view the importance of this result is that it enables us to relate a function $\Phi(t)$ to a transform which has a singularity at the origin like $s^{-\alpha}$ even when $\Re(\alpha)<0$. The essential property of $\Phi(t)$ which made the proof possible was that it satisfied the equation

$$
\frac{d}{d t} F(t, \alpha)=F(t, \alpha-1)
$$

so that successive integrations by parts could be carried out to give (5). Many functions are known to satisfy this " $F$ " equation (7). For example, all the functions $\Phi(t)$ which appear in the table satisfy this equation and the proof of the theorem in each case goes through as in case 1 .

The functions $\Phi(t)$ in the table have been chosen because their transform functions $\phi(s)$ have essentially distinct types of singularities at the origin. In the next section we shall show how even these few choices enable us to handle a considerable variety of Laplace transforms.

Cases 2 and 4 can be obtained formally from cases 1 and 3 respectively by differentiation with respect to the parameter $\alpha$. Case 5 can be obtained formally from case 1 by first integrating with respect to $\alpha$ and then subtracting the effect of the pole at $s=1$. (In this case we restrict our attention to the branch on which $\log s$ is real when $s$ is real.)

From a somewhat different point of view cases 1 and 2 have been considered by Doetsch (4, pp. 494-502).

4. Procedure and examples. We shall now show how the results of the preceding two sections can be used to obtain the asymptotic behaviour of the inverse of a variety of Laplace transforms. We shall consider a few examples 
to illustrate the procedure and then summarize the steps to be taken in any particular case.

We shall first consider the function

$$
f(s)=\frac{\log s}{1+s^{2}} .
$$

This function is a Laplace transform which can be inverted by means of the inversion integral (4, pp. 263-264) and the problem is to obtain the behaviour of its inverse $F(t)$ for large $t$. (In fact $F(t)=\cos t \operatorname{Si}(t)-\sin t \operatorname{Ci}(t)$ but this need not be known in what follows.)

If we subtract from $f(s)$ the effect of the poles at $s= \pm i$ we obtain the function

$$
\frac{\log s}{1+s^{2}}-\frac{\pi s}{2+2 s^{2}}
$$

which satisfies the conditions of Theorem 1 . Therefore

$$
\begin{aligned}
F(t) & -\frac{1}{2} \pi \cos t=\frac{1}{2 \pi i} \int_{C} e^{s t}\left[\frac{\log s}{1+s^{2}}-\frac{\pi s}{2+2 s^{2}}\right] d s \\
& =\frac{1}{2 \pi i} \int_{C} e^{s t}\left[\sum_{j=0}^{n}(-1)^{j} s^{2 j} \log s+\frac{(-1)^{n+1} s^{2 n+2} \log s}{1+s^{2}}-\frac{\pi s}{2+2 s^{2}}\right] d s
\end{aligned}
$$

where $C$ in Fig. 1 may be taken to the left of the points $s= \pm i$.

Now from Theorem 2 (case $2 c$ ) we know that

(8) $-\sum_{j=0}^{n}(-1)^{j}(2 j) ! t^{-2 j-1}=\frac{1}{2 \pi i} \int_{C} e^{s t}\left[\sum_{j=0}^{n}(-1)^{j} s^{2 j} \log s+\right.$ entire function $] d s$.

In each of (7) and (8) contributions to the integrals from $C^{\prime}$, the part of $C$ outside the circle $|s|=r$, are $o\left(e^{-\epsilon t}\right)$ as $t \rightarrow \infty$, for some $\epsilon>0$ because of Theorem 1. Contributions from the remainder of $C$ due to the terms $\pi s /\left(1+s^{2}\right)$ and the entire function are also $o\left(e^{-\epsilon t}\right)$ as $t \rightarrow \infty$ because the contour can be moved, as far as these terms are concerned, to a position lying entirely to the left of the imaginary axis.

We therefore obtain

$F(t)-\frac{1}{2} \pi \cos t+\sum_{j=0}^{n}(-1)^{j}(2 j) ! t^{-2 j-1}=\frac{(-1)^{n+1}}{2 \pi i} \int_{C,|s| \leqslant r} \frac{e^{s t} s^{2 n+2} \log s}{1+s^{2}} d s+o\left(e^{-\epsilon t}\right)$ as $t \rightarrow \infty$. The integral on the right can be divided into two parts: one taken along the rays arg $s= \pm \psi$ and the other taken around a portion of a small circle enclosing the origin. We choose $t$ large and let the radius of this circle be $1 / t$. From the first part we then have

$$
\begin{aligned}
\left|\int e^{s t} \frac{s^{2 n+2} \log s}{1+s^{2}} d s\right| & \leqslant A \int_{1 / t}^{r} e^{u t \cos \psi} u^{2 n+1} d u \\
& =A t^{-2 n-2} \int_{1}^{r t} e^{v \cos \psi} v^{2 n+1} d v \\
& \leqslant A t^{-2 n-2} \int_{1}^{\infty} e^{v \cos \psi} v^{2 n+1} d v \\
& =B t^{-2 n-2}
\end{aligned}
$$


where $A, B$ are finite constants. For the second part we have

$$
\left|\int e^{s t} \frac{s^{2 n+2} \log s}{1+s^{2}} d s\right| \leqslant e^{t(1 / t)}(1 / t)^{2 n+1} 2 \pi(1 / t) .
$$

We therefore obtain finally as $t \rightarrow \infty$.

$$
F(t)=\frac{1}{2} \pi \cos t-\sum_{j=0}^{n}(-1)^{j}(2 j) ! t^{-2 j-1}+O\left(t^{-2 n-2}\right)
$$

As a second example consider

$$
\begin{aligned}
f(s) & =\frac{e^{-1 / s}}{s(1+s)} \\
& =\sum_{j=0}^{n}(-1)^{j} s^{j-1} e^{-1 / s}+\frac{(-1)^{n+1} s^{n} e^{-1 / s}}{1+s} .
\end{aligned}
$$

The procedure for this example is similar to the one given above except in the treatment of the remainder. This time we let the radius of the small circle about the origin be $t^{-\frac{1}{2}}$ so that, for the part of the remainder taken along the rays $\arg s= \pm \psi$, we have

$$
\begin{aligned}
\left|\int \frac{e^{s t} e^{-1 / s} s^{n}}{1+s} d s\right| & \leqslant C \int_{t^{-\frac{1}{2}}}^{r} e^{t u \cos \psi} e^{-\cos \psi / u} u^{n} d u \\
& =C t^{-\frac{1}{2} n-\frac{1}{2}} \int_{1}^{r \vee t} e^{\vee t \cos \psi(v-1 / v)} v^{n} d v \\
& \leqslant D t^{-\frac{1}{2} n-\frac{1}{2}}
\end{aligned}
$$

where $C, D$ are finite constants. For the part of the remainder taken around the small circle we obtain

$$
\left|\int \frac{e^{s t} e^{-1 / s} s^{n}}{1+s} d s\right| \leqslant e^{v} e^{-v t} t^{-\frac{1}{2} n} 2 \pi t^{-\frac{1}{2}}
$$

so that finally

$$
F(t)=\sum_{j=0}^{n} t^{-j / 2} J_{j}(2 \sqrt{ } t)+O\left(t^{-\frac{1}{2} n-\frac{1}{2}}\right)
$$

as $t \rightarrow \infty$.

On the other hand, for

we obtain

$$
f(s)=\frac{e^{1 / s}}{s(1+s)}
$$

$$
F(t)=\sum_{j=0}^{n} t^{-\frac{1}{2} j} I_{j}(2 \sqrt{ } t)+O\left(t^{-\frac{1}{2} n-\frac{1}{2}} e^{+2 \sqrt{ } t}\right)
$$

as $t \rightarrow \infty$.

As a final example let us consider a transform with $\log s$ in the denominator. If

$$
f(s)=\frac{1}{(1-s) \log s}
$$


we obtain, as we did in (7),

$$
\begin{aligned}
F(t) & +\left(\frac{1}{2}+t\right) e^{t} \\
& =\frac{1}{2 \pi i} \int_{C} e^{s t}\left[\sum_{j=0}^{n}\left(\frac{s^{j}}{\log s}-\frac{1}{s-1}\right)+\frac{n+1}{s-1}+\frac{s^{n+1}}{(1-s) \log s}+\frac{1}{2} \frac{s+1}{(s-1)^{2}}\right] d s .
\end{aligned}
$$

Using case 5 of the table and treating the remainder as we did in the first example we obtain finally

$$
F(t)=-\left(\frac{1}{2}+t\right) e^{t}-\sum_{j=0}^{n}\left(\int_{-j}^{\infty} \frac{t^{u-1}}{\Gamma(u)} d u-e^{t}\right)+O\left(t^{-n-2}\right)
$$

as $t \rightarrow \infty$.

It is clear that the above procedure can be applied even when the singularity being considered is not at the origin; a simple change of variable will move the singularity to the origin and multiply the function $F(t)$ by an exponential factor.

In summary, the procedure for finding the asymptotic behaviour of the inverse of a given $f(s)$ can be described briefly as follows. We first verify that the inverse exists and can be found by means of the inversion integral (4, pp. 263-264). We then move the singularity under consideration to the origin, subtract the effect of any poles to the right of, or on, the imaginary axis and verify that the conditions of Theorem 1 are satisfied. We then subtract the effect of a finite number of appropriate terms chosen from the table and finally examine the size of the remainder for large $t$.

The procedure can also be used when we have to consider a finite number of singularities with the same real part; each singularity can be considered separately.

5. Asymptotic behaviour of a certain function. In the first example of the previous section the asymptotic expansion proceeded in negative powers of $t$. A comparable expansion, which will of course contain sine and cosine terms, can be obtained for the examples involving Bessel functions; we have only to substitute the known asymptotic expansions of the Bessel functions and neglect any term whose order is the same as or smaller than the order of the remainder.

To obtain an expansion in terms of elementary functions for the last example we would need an asymptotic expansion for the function

$$
\Phi(t)=\int_{\alpha}^{\alpha+\infty} \frac{t^{u-1}}{\Gamma(u)} d u-e^{t} .
$$

We shall find an expansion of this function but it will turn out that the expansion proceeds in negative powers of $\log t$. It can therefore be substituted into examples like the last one only when the first term in the sum is sufficient.

Because of Theorems 1 and 2 we can write

$$
\Phi(t)=\frac{1}{2 \pi i} \int_{C} \frac{e^{s t}}{s^{\alpha} \log s} d s+o\left(e^{-\epsilon t}\right), \quad t \rightarrow \infty .
$$


Letting the radius of the circular part of $C$ be $1 / t$ and $s=u / t$ we obtain

$$
\Phi(t)=\frac{t^{\alpha-1}}{2 \pi i} \int \frac{e^{u} d u}{u^{\alpha}(\log u-\log t)}+o\left(e^{-\epsilon t}\right), \quad t \rightarrow \infty,
$$

where the integration is taken around a contour like $C$ except that the circle about the origin has radius $=1$.

$$
\begin{aligned}
\Phi(t) & =-\frac{t^{\alpha-1}}{\log t}\left[\frac{1}{2 \pi i} \int \frac{e^{u}}{u^{\alpha}} \sum_{l=0}^{n}\left(\frac{\log u}{\log t}\right)^{l} d u+\frac{1}{2 \pi i} \int \frac{e^{u}}{u^{\alpha}}\left(\frac{\log u}{\log t}\right)^{n+1} \frac{d u}{1-\log u / \log t}\right] \\
& =-\frac{t^{\alpha-1}}{\log t}\left[\sum_{l=0}^{n}\left(\frac{1}{\log t}\right)^{l}\left(-\frac{d}{d \alpha}\right)^{l} \frac{1}{\Gamma(\alpha)}+O\left(\left(\frac{1}{\log t}\right)^{n+1}\right)\right]
\end{aligned}
$$

as $t \rightarrow \infty$. We have used Hankel's contour integral representation of $1 / \Gamma(\alpha)$. Our result includes some special cases considered by Colombo (3).

In case $\Re(\alpha)>-1$

$$
\Phi(t)=\frac{1}{2 \pi i} \int_{C} \frac{e^{s t}}{s^{\alpha} \log s} d s
$$

for all $t>0$. By taking $\psi=\pi$ and shrinking the circular part of $C$ to zero we can obtain a result found by Ramanujan for real $\alpha$ (5, p. 196).

We wish to thank F. M. C. Goodspeed and R. D. James for helpful discussions during the preparation of this paper.

\section{REFERENCES}

1. V. Bush, Operational circuit analysis (New York, 1929).

2. J. R. Carson, Electric circuit theory and the operational calculus (New York, 1926).

3. S. Colombo, Sur quelques transcendantes introduites par la résolution des équations intégrales de Volterra à noyaux logarithmiques, Bull. Sci. Math. (2), 77 (1953), 89-104.

4. G. Doetsch, Handbuch der Laplace-Transformation (Basel, 1950), Bd. 1.

5. G. H. Hardy, Ramanujan (Cambridge, 1940).

6. O. Heaviside, Electromagnetic theory (London, 1899), vol. 2.

7. C. Truesdell, $A$ unified theory of special functions (Princeton, Ann. Math. Studies, no. 18, 1948).

\section{University of British Columbia}

